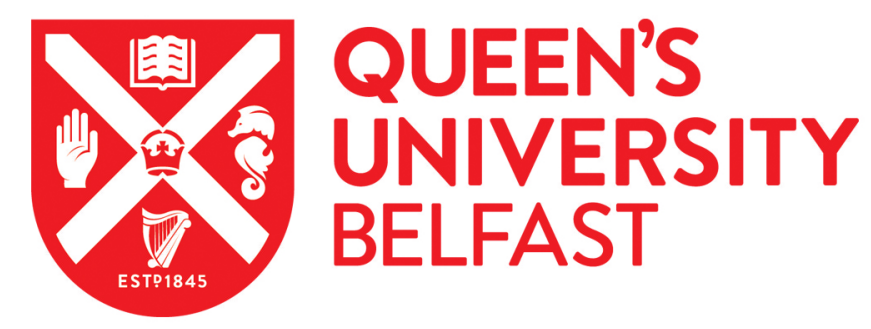

\title{
Cognitively-Inspired Artificial Bee Colony Clustering for Cognitive Wireless Sensor Networks
}

Kim, S-S., McLoone, S., Byeon, J-H., Lee, S., \& Liu, H. (2017). Cognitively-Inspired Artificial Bee Colony Clustering for Cognitive Wireless Sensor Networks. Cognitive Computation, 9(2), 207-224.

https://doi.org/10.1007/s12559-016-9447-z

Published in:

Cognitive Computation

Document Version:

Peer reviewed version

Queen's University Belfast - Research Portal:

Link to publication record in Queen's University Belfast Research Portal

Publisher rights

(C) Springer Science+Business Media New York 2017.

This work is made available online in accordance with the publisher's policies. Please refer to any applicable terms of use of the publisher.

\section{General rights}

Copyright for the publications made accessible via the Queen's University Belfast Research Portal is retained by the author(s) and / or other copyright owners and it is a condition of accessing these publications that users recognise and abide by the legal requirements associated with these rights.

Take down policy

The Research Portal is Queen's institutional repository that provides access to Queen's research output. Every effort has been made to ensure that content in the Research Portal does not infringe any person's rights, or applicable UK laws. If you discover content in the Research Portal that you believe breaches copyright or violates any law, please contact openaccess@qub.ac.uk. 


\title{
Cognitively-Inspired Artificial Bee Colony Clustering for Cognitive Wireless Sensor Networks
}

\author{
Sung-Soo Kim • Seán McLoone • \\ Ji-Hwan Byeon • Seokcheon Lee • \\ Hongbo Liu
}

Accepted: 09 December 2016

\begin{abstract}
The swarm cognitive behavior of bees readily translates to swarm intelligence with "social cognition", thus giving rise to the rapid promotion of survival skills and resource allocation. This paper presents a novel cognitivelyinspired artificial bee colony clustering (ABCC) algorithm with a clustering evaluation model to manage the energy consumption in cognitive wireless sensor networks (CWSNs). The ABCC algorithm can optimally align with the dynamics of the sensor nodes and cluster heads in CWSNs. These sensor nodes and cluster heads adapt to topological changes in the network graph over time. One of the major challenges with employing CWSNs is to maximize the lifetime of the networks. The ABCC algorithm is able to reduce and balance the energy consumption of nodes across the networks. Artificial Bee Colony $(\mathrm{ABC})$ optimization is attractive for this application as the cognitive behaviors of artificial bees match perfectly with the intrinsic dynamics
\end{abstract}

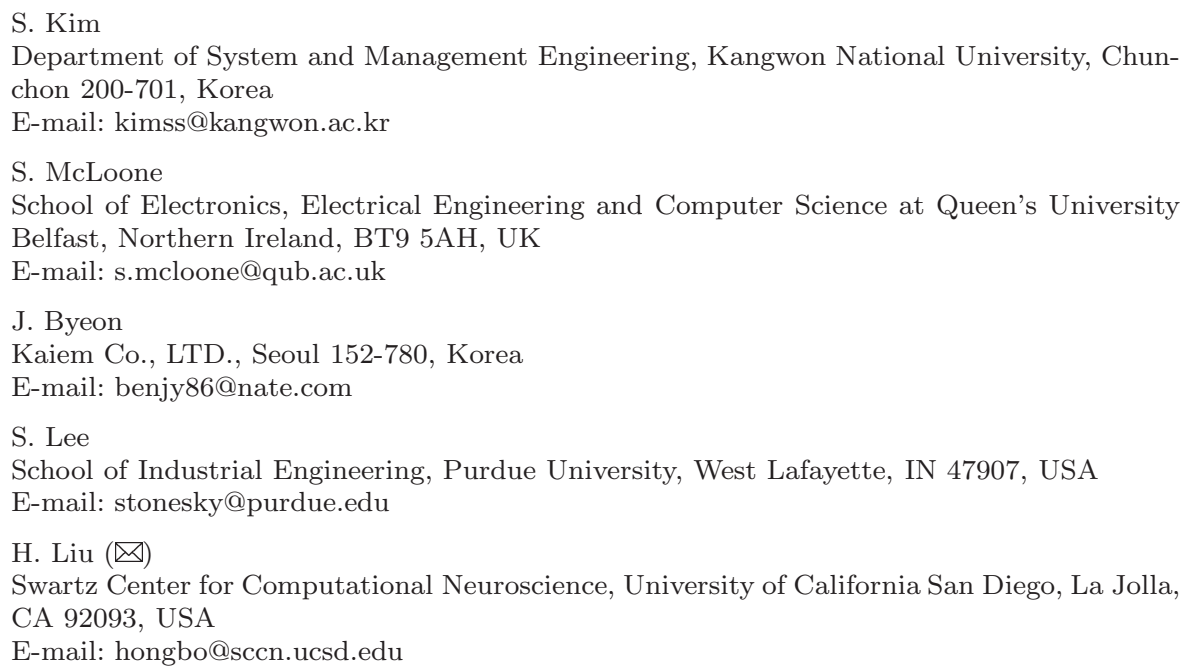


in cognitive wireless sensor networks. Additionally, it employs fewer control parameters compared to other heuristic algorithms, making identification of optimal parameter settings easier. Simulation results illustrate that the ABCC algorithm outperforms PSO (particle swarm optimisation), GSO (group search optimisation), LEACH (low-energy adaptive clustering hierarchy), LEACH-C (LEACH-centralized), and HEED (hybrid energy-efficient distributed clustering) for energy management in CWSNs. Our proposed algorithm is increasingly superior to these other approaches as the number of nodes in the network grows.

Keywords Cognitive Wireless Sensor Network (CWSN) · Clustering • Artificial Bee Colony $(\mathrm{ABC}) \cdot$ Clustering evaluation model

\section{Introduction}

Human beings possess high-level social cognition. In fact, swarm behaviors already widely exist in other social creatures with low-level cognition such as bees and ants. It is straightforward to associate swarm intelligence with "social cognition" that leads to the rapid promotion of survival skills and resource allocation. There have been major advances in swarm intelligence based cognitively-inspired algorithms [8,31]. He et al. [9] reported an group search optimization (GSO) algorithm inspired by animal behaviors, especially animal searching behaviors. Bishop proposed a search algorithm with stochastic diffusion characteristics [4,28], and Kennedy and Eberhart [17] proposed particle swarm optimization (PSO) algorithm mainly inspired by social behaviour patterns of organisms that live and interact within large groups. In particular, PSO incorporates swarming behaviours observed in flocks of birds, schools of fish, or swarms of bees, and even human social behavior. al-Rifaie et al. [29] introduced a novel hybrid algorithm mimicking the behavior of birds flocking and ants foraging. Dubey et al. [7] proposed a cognitively-inspired modified flower pollination algorithm. Their results illustrated that these algorithms had some advantages in terms of cost and statistical performance. Ullah et al. [33] proposed a cognitively-inspired soft switching approach to implement horizontal cloud elasticity. It could reduce the likelihood of oscillation and increase the stability of the underlying cloud computing system. Kim et al. [18] developed a discrete $\mathrm{ABC}$ algorithm for binary integer job scheduling problems in grid computing. They illustrated that the performance of efficient binary ABC was better than alternatives such as genetic algorithms, simulated annealing, and particle swarm optimization. Ye and Chen [35] proposed an efficient and competitive combinatorial $\mathrm{ABC}$ algorithm for solving the minimum attribute reduction problem. The discrete version of $\mathrm{ABC}$ outperformed all competing approaches in terms of solution quality. The ABC algorithm can also be used in the emerging area of big data analytics. Ding et al. [6] have observed that achieving cognition ability when clustering big data is a major challenge in stream data mining, while Abdullah et al. [2], who introduce a special issue on the topic, note the potential of cognitive computing systems that are trained 
using biologically inspired approaches and computational intelligence. In this paper, we present a novel Artificial Bee Colony Clustering (ABCC) algorithm with a clustering evaluation model to manage the energy consumption in cognitive wireless sensor networks.

Cognitive wireless sensor networks (CWSNs) are an emerging technology with the potential to address traditional wireless network problems such as reliability. There are three main types of routing model employed in cognitive wireless sensor networks, namely the direct transmission (one hop) model, the multi-hop model, and the cluster-based hierarchical model, as shown in Figure 1 [13]. Due to the potential for infeasible solutions with the direct transmission (one hop) model and the latency associated with the multi-hop model, the cluster-based hierarchical model is considered the most promising. A generic CWSN architecture is illustrated in Figure 2. Sensor nodes are the core components with clusters of nodes acting as organizational units. Each cluster has a cluster head as its organizational leader. There are multiple cluster-based hierarchical levels (CH-levels). The base station (Sink) is at the upper level of the hierarchical CWSN. A major constraint is the energy requirements of each node, which ultimately determine network lifetime.

Abbasi and Younis [1] surveyed clustering algorithms for cognitive wireless sensor networks (CWSNs). Designing and operating large networks would require scalable architectural and management strategies. In addition, sensors in such large scale deployments are generally energy constrained as their batteries cannot be recharged. Since each wireless sensor node only has limited energy storage, the efficient use of this energy is vital to extend network lifetime, and hence the range of suitable applications for these networks. Appropriate clustering of nodes can reduce the overall energy usage in a network. Minimizing energy consumption is an important consideration when developing clustering schemes [5].

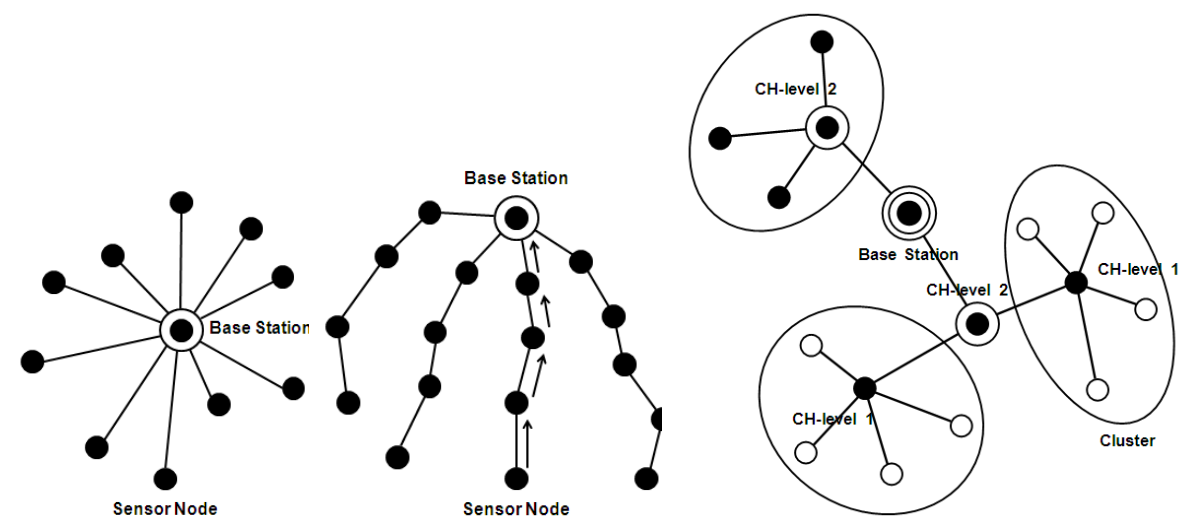

(a) Direct transmission (b) Multi-hop model

(c) Cluster-based hierarchical model (One hop model)

Fig. 1 Routing model 


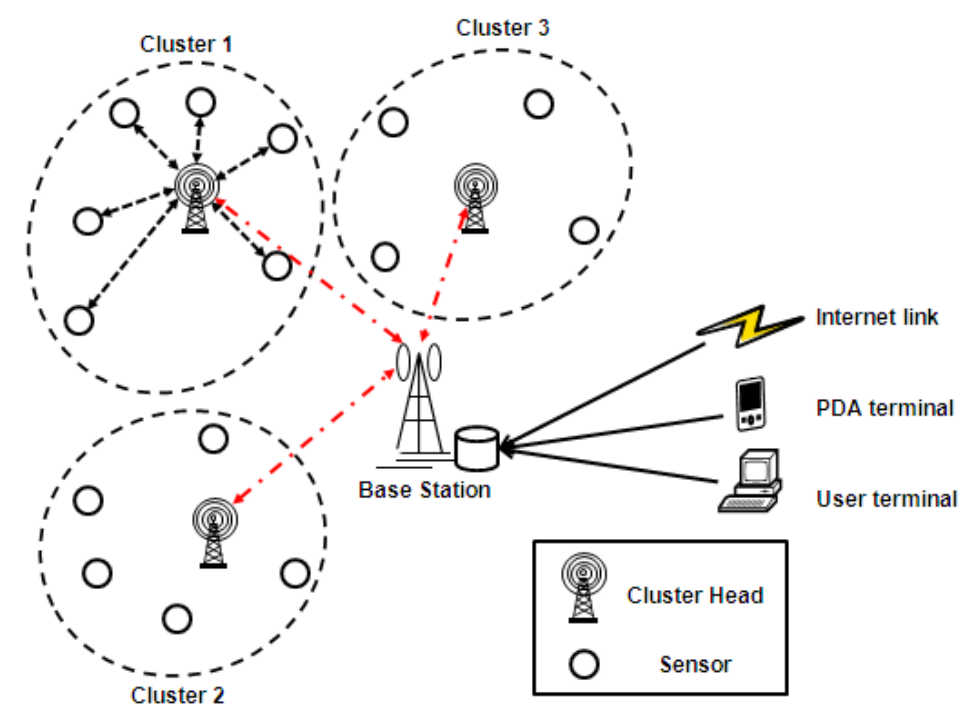

Fig. 2 Cognitive Wireless Sensor Network Architecture

Clustering in CWSNs has proven to be an effective approach to organizing a network into a connected hierarchy, so that it balances the load and prolongs network lifetime. However, this involves grouping nodes into clusters and electing a cluster head, with the collection of cluster heads in the network forming a connected dominating set. Since obtaining an optimal dominating set is an NP-complete problem, the proposed algorithms are heuristic in nature [37]. Kulkarni [19] provided an extensive survey of computational intelligence applications to various problems in wireless sensor networks. They concluded that swarm intelligence with cognitive behaviors $[22,25]$ was most appropriate for the design and deployment of sensor networks.

A cognitive wireless sensor network is an intelligent wireless communication paradigm that is dynamically aware of its surrounding environment. It is also able to respond to it in order to achieve reliable and efficient communication. The dynamical cognition capability and environmental adaptability rely on organizing dynamical networks effectively. However, as already noted, optimally clustering the cognitive wireless sensor networks is an NP-complete problem. In this paper, we present an Artificial Bee Colony Clustering (ABCC) algorithm for optimally configuring cognitive wireless sensor networks. Our basic idea is to use the cognitive behavior of bees in our ABCC algorithm to mimic the dynamical cognition capability and environmental adaptability of CWSNs. One of the main merits of the combination of $\mathrm{ABC}$ and clustering in such a heuristic way is that it can determine dynamically the optimal number and assignment of cluster heads in CWSNs, with each sensor node then connecting to its nearest cluster head. Furthermore, it also optimally coincides with the dynamics of the cluster heads and sensor nodes, which can adapt to topological changes to any network graph over time. We further formulate the clustering evaluation 
model and use it as the basis for an objective function. It simultaneously minimizes the average dissipated energy and the standard deviation of the residual energy. To demonstrate the efficacy of ABCC, we compare its performance to that of a number of competing approaches, specifically particle swarm optimisation (PSO), group search optimisation (GSO), the low-energy adaptive clustering hierarchy (LEACH), LEACH-centralized (LEACH-C) [10,11], and the hybrid energy-efficient distributed clustering (HEED) [36] algorithms.

The major contributions of this paper are as follows:

- We formulate the clustering problem for CWSNs with the objective of simultaneously minimizing the average dissipated energy and the standard deviation of residual energy of nodes to prolong network lifetime.

- A cognitively-inspired Artificial Bee Colony Clustering (ABCC) algorithm is presented, in which the cognitive behaviors of artificial bees match perfectly with the intrinsic dynamics in CWSNs.

- We develop an evaluation model as an objective function that considers the dissipated energy to conserve and balance energy consumption of nodes in CWSNs.

The remainder of the paper is organized as follows. Section 2 describes related work. Section 3 discusses some theoretical formulations related to the integer programming clustering model applied to the CWSN. In Section 4, we present our ABCC for CWSNs in detail. Experimental results, comparisons and discussions are provided in Section 5 and finally conclusions are presented in Section 6.

\section{Related Work}

Heinnzelman et al. $[10,11]$ proposed LEACH, a clustering-based protocol that utilizes randomized rotation of cluster-heads to evenly distribute the energy load among the sensors in the network. In their experiments, LEACH is shown to reduce energy dissipation and increase network lifetime compared with conventional routing protocols. Heinnzelman et al. [10] showed that LEACH can achieve good performance in terms of system lifetime, latency, and applicationperceived quality. They also developed LEACH-C, a protocol that uses a centralized clustering algorithm and the same steady-state protocol as LEACH. The base station (BS) computes the average node energy, and nodes that have energy below this average are not allowed to be cluster heads. In LEACH, every node has to be reachable in a single hop, and the load distribution is uniform among all nodes [37]. LEACH assigns a fixed probability (5\%) to every node of electing itself as a cluster head. Salim et al. [30] proposed a IBLEACH (clustering routing protocol called intra-balanced LEACH) algorithm, which extends the LEACH protocol by balancing energy consumption in the network. They elect the non-optimal cluster heads randomly from all nodes during the setup phase in a similar fashion to LEACH. Aslam et al. [3] presented energy efficient hierarchical routing protocols developed from the conventional LEACH routing protocol to increase lifetime. 
Liu [23] presented a survey on clustering based routing protocols, outlining their advantages. They also highlighted that further research is needed to determine how to handle the overhead of node mobility and topology changes. Younis and Fahmy [36] proposed HEED that periodically selects cluster heads according to a hybrid of the node residual energy and an overhead. It can achieve fairly uniform cluster head distribution across networks. They demonstrated that their proposed approach was effective in prolonging network lifetime and supporting scalable data aggregation. In the HEED protocol all nodes are assumed to be equally important [37]. A node uses its residual energy as the primary parameter to probabilistically elect itself to become a cluster head. Thus, the LEACH and HEED clustering methods both have a limitation in that they do not identify or use the optimal number of cluster heads at each stage.

Okdem et al. [26] presented performance tests and complexity analysis of cluster based wireless sensor network routing based on an artificial bee colony (ABC) algorithm. Performance and analysis results showed that the ABC algorithm presented promising solutions. Karaboga et al. [15] also considered the ABC algorithm for cluster based wireless sensor network routing. They [16] proposed an ICWAQ (Improved version of Cluster based Wireless sensor network routings using Artificial bee colony algorithm considering Quality of service) algorithm to optimize clustering of the nodes during the cluster-heads selection process. These heuristic methods also have the limitation that the objective function is mainly based on the distance between nodes as a metric to encourage energy reduction.

\section{Clustering Evaluation Model}

The dense deployment and unattended nature of cognitive wireless sensor networks make it quite difficult to recharge node batteries. Therefore, energy efficiency is very important in these networks. Clustering can improve network lifetime, a primary metric for evaluating the performance of a sensor network. Periodic re-clustering is necessary in order to heal disconnected regions and to distribute energy consumption across all nodes [37].

We assume that a network consists of $N$ nodes, and that $E_{t}(n)_{\text {dissipate, as }}$ defined in Equ. (1), is the energy dissipated in the cluster head node $(n \in C H)$ or non-cluster head node $(n \in$ non $-C H)$ in the network during a single round $t$. Here, $l$ is the number of bits in each data message and $d_{n t o B S}$ is the distance from the cluster head node to the base station $B S$. Each cluster head dissipates energy receiving signals from the nodes, aggregating the signals, and transmitting the aggregate signal to the base station $(B S)$, which is a Sink. Since $B S$ is far from the nodes, it is assumed that the energy dissipation follows the multipath model ( $d^{4}$ power loss). Each non-cluster head node only needs to transmit its data to the cluster head once during a round. The distance from non-cluster head nodes to the cluster head is assumed to be small so that the energy dissipation follows the Friss free-space model ( $d^{2}$ power loss). 
The distance from the node to the cluster head is denoted as $d_{n t o C H}$, and $m_{n}$ is the actual number of sensor nodes that are connected to cluster head node $n$. The communication energy parameters are the initial energy for the nodes $E_{j}$, radio electronics dissipation for receiving and transmitting units $E_{\text {elec }}$, amplifier energy parameters $E_{m p}$ and $E_{f s}$, energy for data aggregation $E_{D A}$, and the number of bits in each data message $l=1024 b i t s[10,11]$.

We use Equ. (1) to get $E_{t}(n)_{\text {dissipate }}$ for each cluster head node $(n \in C H)$ and each non-cluster head node $(n \in$ non $-\mathrm{CH})$. The sum of the dissipated energy for round $t$ is calculated using Equ. (2). This is a binary integer programming model with binary decision variable $x_{n}=1$ for each cluster head and $x_{n}=0$ for each sensor node $n$, as defined in Equ. (3). Each sensor node member is connected to its closest cluster head once the cluster heads have been identified. Our objective is to minimize: (1) the average dissipated energy, $A V G\left(E_{\text {dissipate }}\right)$, as expressed in Equs. (2)-(4) to conserve energy, and; (2) the standard deviation of the residual energy, $\operatorname{STDEV}\left(E_{\text {residual }}\right)$, as defined in Equs. (5)-(7) to balance the energy load across all nodes.

$$
E_{t}(n)_{\text {dissipate }}=\left\{\begin{array}{cr}
\left(m_{n}-1\right) l E_{\text {elec }}+m_{n} l E_{D A} & \\
+l E_{\text {elec }}+l E_{m p} d_{n t o B S}^{4} & n \in C H \\
l E_{\text {elec }}+l E_{f s} d_{n t o C H}^{2} & n \in \text { non }-C H
\end{array}\right.
$$

The sum of the dissipated energy for round $t$ is given by:

$$
\begin{gathered}
\sum_{n \in N} E_{t}(n)_{\text {dissipate }}=\sum_{n \in C H} E_{t}(n)_{\text {dissipate }}+\sum_{n \in \text { non }-C H} E_{t}(n)_{\text {dissipate }} \\
=\sum_{n \in N} x_{n}\left[\left(m_{n}-1\right) l E_{\text {elec }}+m_{n} l E_{D A}+l E_{\text {elec }}+l E_{m p} d_{n t o B S}^{4}\right] \\
+\sum_{n \in N}\left(1-x_{n}\right)\left[l E_{\text {elec }}+l E_{f s} d_{n t o C H}^{2}\right]
\end{gathered}
$$

where the decision variable $x_{n}$ for node $n$ is defined as:

$$
x_{n}=\left\{\begin{array}{l}
1 \text { if node } n \text { is cluster head } \\
0 \text { otherwise }
\end{array}\right.
$$

The average dissipated energy for round $t$ is then computed as:

$$
A V G\left(E_{\text {dissipate }}\right)=\left(\sum_{n \in N} E_{t}(n)_{\text {dissipate }}\right) / N
$$

Defining the residual energy for round $(t+1)$ as:

$$
E_{t+1}(n)_{\text {residual }}=E_{t}(n)_{\text {residual }}-E_{t}(n)_{\text {dissipate }}
$$


the average residual energy for round $t$ can be computed as:

$$
A V G\left(E_{\text {residual }}\right)=\sum_{n \in N} \frac{E_{t}(n)_{\text {residual }}}{N}
$$

and the standard deviation of the residual energy is expressed as:

$$
\operatorname{STDEV}\left(E_{\text {residual }}\right)=\sqrt{\sum_{n \in N} \frac{\left(A V G\left(E_{\text {residual }}\right)-E(n)_{\text {residual }}\right)^{2}}{N}}
$$

The objective function in Equ. (8) is used to evaluate the performance of a cognitive wireless sensor network. This function is a weighted combination of the average dissipated energy in Equ. (4) and the standard deviation of residual energy in Equ. (7).

$$
\text { Minimize } A V G\left(E_{\text {dissipate }}\right)+\alpha \times S T D E V\left(E_{\text {residual }}\right)
$$

As an example, consider a network with 15 nodes (i.e. $N=15$ ) with node positions as shown in Table 1 . The sink node is at position $(0,0)$. The binary solution for the network is listed in Table 2. A clustering design model with 6 selected cluster heads (nodes 1, 4, 5, 6, 7 and 13) is illustrated in Figure 3. These cluster heads are connected to the sink node. Sensor nodes 2 and 3 are connected to their closest cluster head (node 4). In a similar fashion, sensor nodes $8,9,10,12$ and 14 are connected to cluster head node 7, and sensor nodes 11 and 15 are connected to cluster head node 13. To compare our simulation results with LEACH, LEACH-C, and HEED [10], we use the following initial communication energy parameters: $E_{j}=0.5 \mathrm{~J}$, $E_{\text {elec }}=50 \mathrm{~nJ} / \mathrm{bit}, E_{m p}=0.0013 \mathrm{pJ} / \mathrm{bit} / \mathrm{m}^{4}, E_{f s}=10 \mathrm{pJ} / \mathrm{bit} / \mathrm{m}^{2}, E_{D A}=$ $5 \mathrm{~nJ} /$ bit/signal, and $l=1024$ bits. The weighting $\alpha$ of the standard deviation of residual energy term in Equ. (8) is set to 1. The residual energy of rounds $22-23$ and dissipated energy of round 22 for this network are shown in Table 3. Here, $A V G\left(E_{\text {dissipate }}\right)=0.000106, A V G\left(E_{\text {residual }}\right)=0.497301$, $S T D E V\left(E_{\text {residual }}\right)=0.000158$, and the objective value is 0.000264 for the

\begin{tabular}{|c|c|c|c|c|c|c|c|c|c|c|c|c|c|c|c|c|}
\hline Index & Sink & 1 & 2 & 3 & 4 & 5 & 6 & 7 & 8 & 9 & 10 & 11 & 12 & 13 & 14 & 15 \\
\hline$x$ & 0 & 65 & 95 & 100 & 75 & 60 & 45 & 40 & 50 & 75 & 45 & 20 & 35 & 10 & 50 & 25 \\
\hline$y$ & 0 & 5 & 10 & 30 & 15 & 20 & 45 & 60 & 85 & 90 & 95 & 80 & 65 & 80 & 100 & 90 \\
\hline
\end{tabular}
15-node network configuration in Figure 3.

Table 1 Position of the 15 nodes in the example network depicted in Figure 3. 
Table 2 Binary solution: decision variable $x_{n}=0$ for a sensor node and $x_{n}=1$ for cluster head node

\begin{tabular}{|c|c|c|c|c|c|c|c|c|c|c|c|c|c|c|c|c|}
\hline Index & node $n$ & 1 & 2 & 3 & 4 & 5 & 6 & 7 & 8 & 9 & 10 & 11 & 12 & 13 & 14 & 15 \\
\hline$x_{n}$ & & 1 & 0 & 0 & 1 & 1 & 1 & 1 & 0 & 0 & 0 & 0 & 0 & 1 & 0 & 0 \\
\hline
\end{tabular}

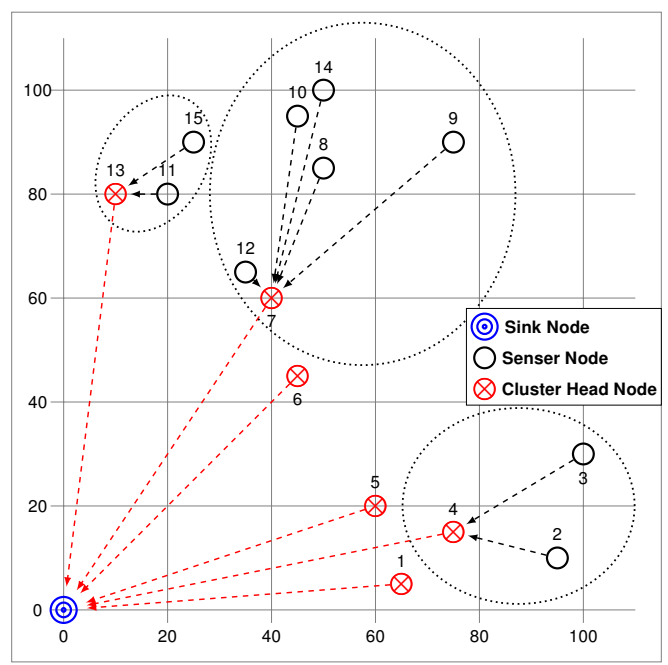

Fig. 3 Clustering design model for a 15-node network with sink node $(0,0)$

Table 3 Residual and dissipated energy of rounds 22-23 in the 15-node network

\begin{tabular}{cccc}
\hline No. of node & $E_{22}(n)_{\text {residual }}$ & $E_{22}(n)_{\text {dissipate }}$ & $E_{23}(n)_{\text {residual }}$ \\
\cline { 1 - 3 } \cline { 3 - 4 } 1 & 0.497465 & 0.000080 & 0.497385 \\
2 & 0.497564 & 0.000055 & 0.497509 \\
3 & 0.497496 & 0.000060 & 0.497436 \\
4 & 0.497616 & 0.000214 & 0.497402 \\
5 & 0.497552 & 0.000078 & 0.497474 \\
6 & 0.497548 & 0.000078 & 0.497470 \\
7 & 0.497430 & 0.000374 & 0.497056 \\
8 & 0.497127 & 0.000058 & 0.497069 \\
9 & 0.497501 & 0.000073 & 0.497428 \\
10 & 0.497388 & 0.000064 & 0.497324 \\
11 & 0.497266 & 0.000052 & 0.497214 \\
12 & 0.497264 & 0.000051 & 0.497213 \\
13 & 0.497251 & 0.000225 & 0.497026 \\
14 & 0.497399 & 0.000069 & 0.497330 \\
15 & 0.497242 & 0.000054 & 0.497188 \\
\hline
\end{tabular}




\section{Methodology}

We propose an Artificial Bee Colony Clustering (ABCC) algorithm that takes advantage of the favourable dynamic properties of artificial bee colonies. It requires fewer control parameters than alternative heuristic methods. In particular, the proposed $\mathrm{ABCC}$ algorithm is optimally designed to deal with the dynamics of the topological changes to any network graph using the model presented in Section 3, with the objective of conserving energy and balancing the energy load across sensor nodes.

\subsection{Artificial Bee Colony}

The artificial bee colony ( $\mathrm{ABC}$ ) is a swarm intelligence algorithm motivated by the cognitive behavior exhibited by honeybees when searching for foods $[24,34]$. The performance of $\mathrm{ABC}$ is better than or similar to other population-based algorithms with the advantage of employing fewer control parameters [20,38]. The control parameter in ABC is Limit, the number of unsuccessful trials before a food source is deemed to be abandoned, with the number of food sources denoted as $S N[18,21,27]$.

In $\mathrm{ABC}$, the colony of artificial bees contains three groups of bees: employed bees, onlooker bees and scout bees. For every food source there is only one employed bee. A fitness function is used to assign a quality or 'nectar' value to the food sources. Each employed bee searches for a new food source within its own neighbourhood and moves to it if it has a higher nectar value. Employed bees then share their food source information (location and nectar value) with the onlooker bees waiting in the hive. Each onlooker bee then selects one of the employed bee food sources probabilistically in a process similar to roulette wheel selection. After selecting its food source, each onlooker bee seeks out one new food source within its neighborhood and moves to this food source if it has a higher nectar value. If the number of active food sources outnumbers the maximum allowed, those with the lowest nectar values are abandoned. An employed bee for a food source that has been abandoned becomes a scout bee and starts to search for a new food source randomly. Thus, while onlooker bees and employed bees are targeted at exploitation, scout bees provide a mechanism for exploration [14]. The flowchart for the ABC algorithm is presented in Figure 4.

\subsection{Artificial Bee Colony Clustering in Cognitive Wireless Sensor Networks}

The cognitive network $(\mathrm{CN})$ is a new type of data network designed to solve some of the issues faced by current networks. The system learns from past experiences (situations, plans, decisions, actions) and uses this knowledge to improve decision making in subsequent machine learning, knowledge representation, computer network, and network management tasks [32]. Hunt et 


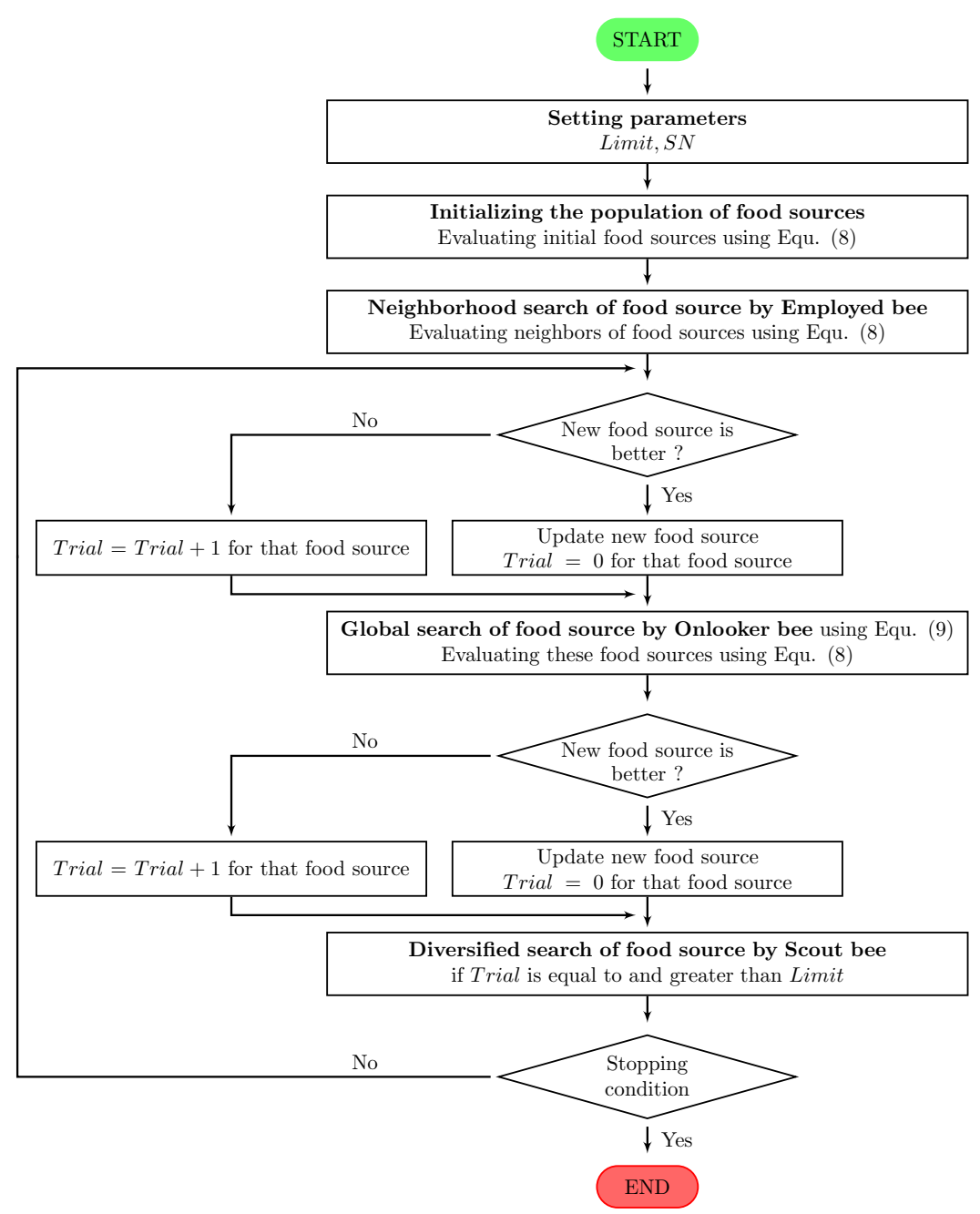

Fig. 4 Flowchart of the ABC algorithm

al. [12] introduced a consensus-based grouping algorithm for multi-agent cooperative task allocation, in which the cognitive behaviors of social animals (bees and ants) are available for cooperation and improved assignments. al-Rifaie et al. [29] also proposed a hybrid-swarm algorithm (mimicking the behaviors of ants and birds) and explored the possibility of it exhibiting computational creativity. In this section, we present a novel artificial bee colony clustering (ABCC) algorithm to obtain the optimal clustering design for cognitive wireless sensor networks. It takes advantage of the cognitive behaviors of artificial bee cognitive systems. Our algorithm seeks to optimize the number and allocation of cluster heads and sensor nodes. The objective is to adaptively cluster 


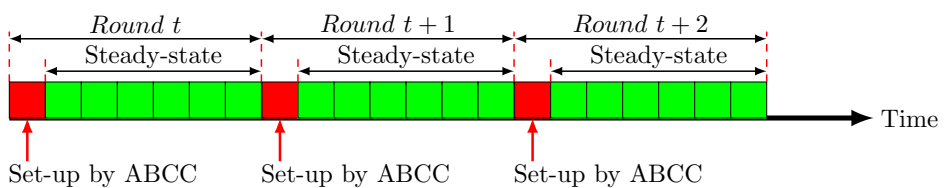

Fig. 5 Time line of clustering design

the cognitive wireless sensor networks in order to conserve energy and balance the energy consumption of nodes in the current round by using active nodes with residual energy. In our artificial bee colony clustering algorithm, the colony of artificial bees also contains three groups of bees: employed bees, onlooker bees and scout bees. For every food source there is only one employed bee, and each food source corresponds to a cluster head. We assign the food sources of the employee bees to the onlooker bees. The onlooker bees help to identify a better cluster head within each cluster (a cluster consists of one cluster head and many sensor nodes), i.e. produce neighbors (new food sources) by probabilistically changing from cluster head to sensor node. Such a hierarchical search strategy matches perfectly with the intrinsic dynamics in cognitive wireless sensor networks. Additionally, in order to avoid the search system becoming rigescent, scout bees generate a new food source (solution) randomly. The cognitive interactions between these three kinds of bee prompt the whole colony to achieve a good solution.

In our artificial bee colony clustering algorithm, the optimal clustering design is divided into rounds in a fashion similar to LEACH. Each round (i.e. $t, t+1, t+2, \cdots)$ is composed of set-up and steady-state phases as shown in Figure 5. There is a set-up phase each time, where the clusters have to be determined [10]. The cluster heads and clusters are organized using our proposed ABCC algorithm in the set-up phase of each round. After the set-up phase, network data is transferred from the nodes to the cluster head, and then to the base station in the time line, and this is referred to as the steady-state phase.

The steps of the Artificial Bee Colony Clustering (ABCC) algorithm, as set out in Algorithm 1, are further explained as follows. The algorithm begins by loading the position data of $N$ nodes in the network and pre-computing the distance between each node, $d_{n t o C H}$, and the distance between each node and the sink node, $d_{n t o B S}$. The initial positions of the nodes are generated randomly in this paper. The network communication energy parameters, namely, the initial energy for the nodes $E_{j}$, the radio electronics energy dissipation for the receiving and transmitting units $E_{\text {elec }}$, amplifier energy parameters $E_{m p}$ and $E_{f s}$, energy of data aggregation $E_{D A}$, and the number of bits in each data message $l=1024 b i t s[10,11]$, are also loaded.

The parameters Limit (maximum number of unsuccessful trials when searching for an improved food source in the vicinity of an active food source) and $S N$ (number of active food sources, employed bees and onlooker bees) for the 


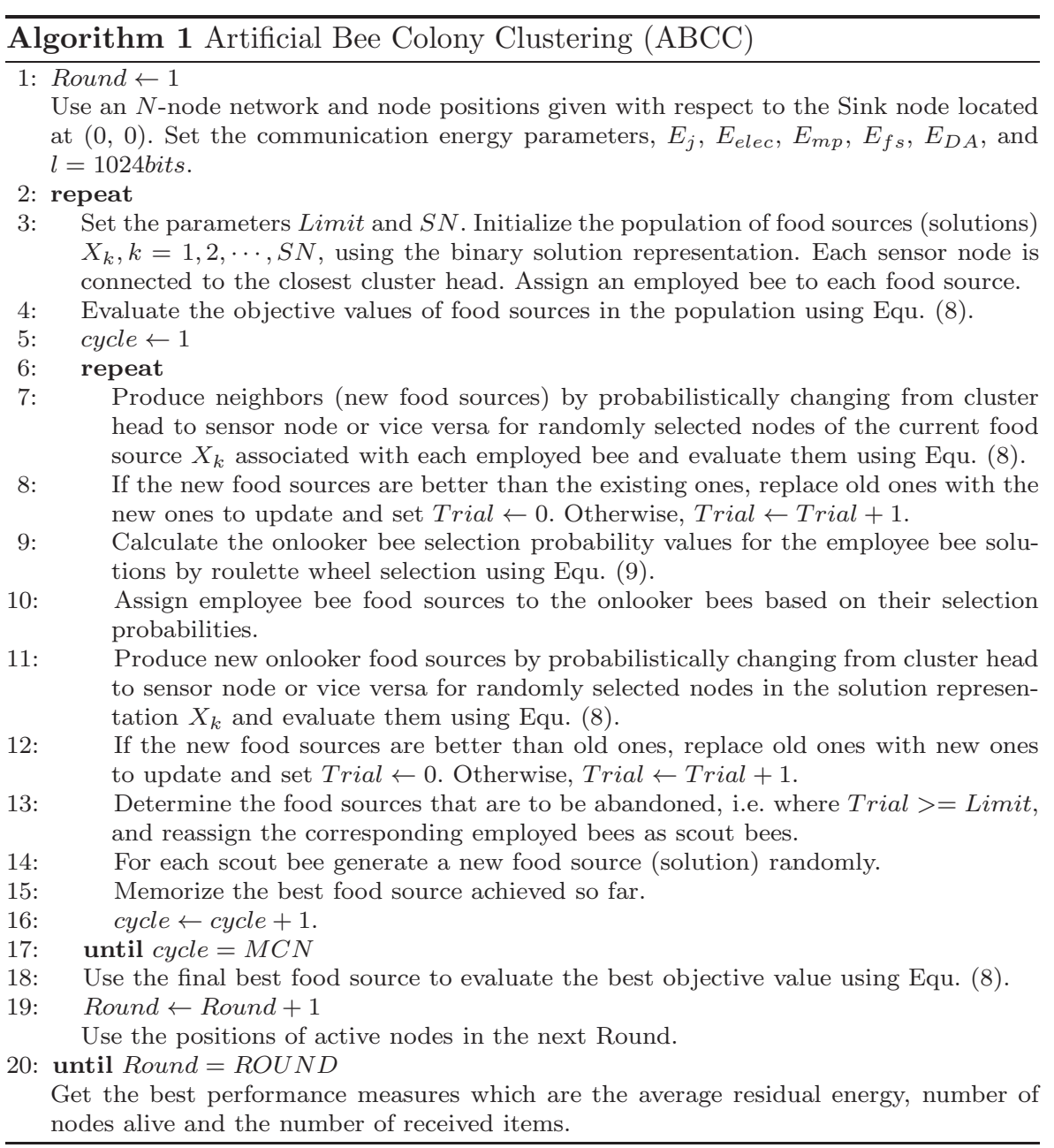

proposed ABCC algorithm are also initialized as is the weighting factor $\alpha$ in Equ. (8).

The population of $S N$ food sources (solutions) are initialized randomly using the binary solution representation, and each node is selected randomly as either ' 1 ' for cluster head or ' 0 ' for sensor node. Each sensor node is then assigned (connected) to its closest cluster head. For example, the network discussed in Section 3 is a food source consisting of 6 cluster heads and 9 connected sensor nodes as shown in Table 2 and Figure 3.

The food sources of the initial population are evaluated using the proposed evaluation function Equ. (8), which is a function of the energy dissipated in the network during a single round $t$. The reciprocal of the evaluation value for food source $k$ (i.e. the output of Equ. (8)) is denoted as $f_{k}$. 
A new neighborhood of each current food source is produced by probabilistically converting a cluster head to a sensor node or vice versa for a randomly selected node.

The new food source is evaluated using Equ. (8). A greedy selection process is then applied for the employed bees, whereby the old food source is replaced by the new one and the Trial count variable is set to ' 0 ' if the new neighborhood food source is better than the old one. Otherwise, the Trial count is increased by 1 .

The onlooker bees then select their food sources based on the evaluation values of the food sources shared by the employed bees. An onlooker bee selects a food source depending on its winning probability value, as defined in Equ. (9), using a roulette wheel selection process.

$$
p_{i}=f_{i} / \sum_{n=1}^{S N} f_{n}
$$

New food sources are then generated for the onlooker bees by probabilistically changing cluster heads to sensor nodes or vice versa, for randomly selected nodes, based on the winning probability of the food sources they have selected. The new food sources are evaluated using the evaluation function (i.e. Equ. (8)) and greedy selection employed by the onlooker bees.

Finally, if abandoned food source exists (i.e. if the Trial count variable exceeds the Limit value), the corresponding employee bees are re-assigned as scout bees and new food sources are also generated for them randomly.

The procedure is repeated until a predetermined maximum number of cycles $(M C N)$ is reached, with the best food source achieved during each cycle memorized. The overall best food source identified by ABCC is selected as the final clustering solution, defining the cluster heads and associated sensor nodes to be employed in this round.

For the next round the network is defined as the positions of active nodes and their associated communication energy parameters, that is to say, the nodes which have expired (run out of energy) during the current round are deleted from the network for subsequent rounds. Rounds continue until the maximum round $(R O U N D)$ is reached, at which point the last node has died.

The current 'state of health' of the CWSN is monitored by tracking the average residual energy, number of active (living) nodes and number of received items at a given round, while the overall performance is captured by the first node failure, the network lifetime and the total amount of transmitted data.

\section{Experiments and Analysis}

\subsection{Experimental Settings}

To illustrate the effectiveness and performance of the proposed ABCC algorithm, we test and compare its performance with a number of compet- 


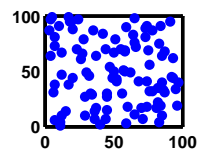

(a) 100 nodes

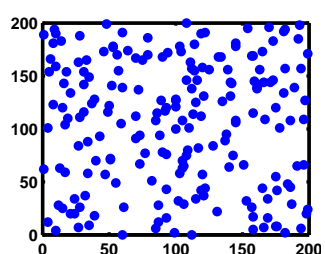

(b) 200 nodes

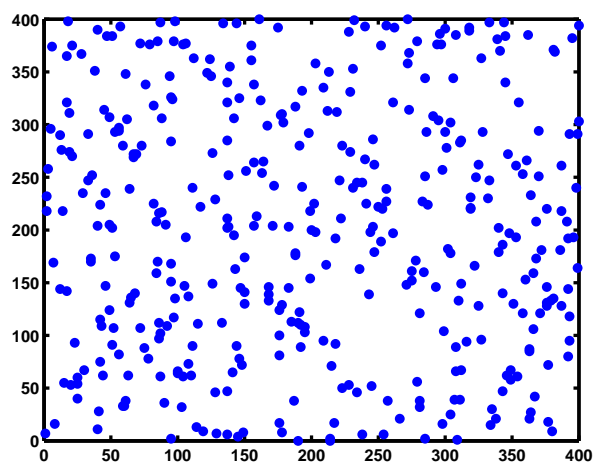

(c) 400 nodes

Fig. 6 Locations of 100, 200, and 400 nodes at Round 1

ing clustering design protocols, namely, particle swarm optimisation (PSO), group search optimisation (GSO), low-energy adaptive clustering hierarchy (LEACH), LEACH-centralized (LEACH-C), and the hybrid energy-efficient distributed clustering (HEED) algorithms. The parameter values for these approaches are selected in accordance with the values specified in [9-11,22,36]. We randomly generate networks with $N=100,200$, and 400 nodes for two scenarios, one with the sink node located at $(0,0)$ and the other with the sink node located at the centre of the network. The positions of nodes are randomly generated for each network on $100 \mathrm{~m} \times 100 \mathrm{~m}, 200 \mathrm{~m} \times 200 \mathrm{~m}$, and $400 \mathrm{~m} \times 400 \mathrm{~m}$ square grids, respectively, as shown in Figure 6 . The initial values of the communication energy parameters for the networks were set as $E_{j}=0.5 \mathrm{~J}$, $E_{\text {elec }}=50 \mathrm{~nJ} / \mathrm{bit}, E_{m p}=0.0013 \mathrm{pJ} / \mathrm{bit} / \mathrm{m}^{4}, E_{f s}=10 \mathrm{pJ} / \mathrm{bit} / \mathrm{m}^{2}, E_{D A}=$ $5 \mathrm{~nJ} / \mathrm{bit} / \mathrm{signal}$, and $l=1024 \mathrm{bits}$, as given in $[10,11]$. The experiments were run on an Intel ${ }^{\circledR}$ Core ${ }^{\mathrm{TM}_{2}}$ Duo CPU $(2.66 \mathrm{GHz}, 2 \mathrm{G}$ RAM). The best parameter values for the $\mathrm{ABCC}$ algorithm were determined experimentally to be $S N=20$ and Limit $=100$.

\subsection{Comparisons to related swarm intelligent algorithms}

Since PSO and GSO are optimisation algorithms, we can use them as alternatives to ABC with our clustering evaluation model (introduced in Section 3) to solve the CWSN clustering problem. These implementations will be denoted as 'PSOC' and 'GSOC', respectively.

The rate of convergence of the PSOC, GSOC and ABCC algorithms in Round 1 for each configuration is illustrated in Figure 7. As expected, ABCC consistently converges as the number of generations increases. It is able to find a stable solution in under 1000 generations in all cases. The results of PSOC, GSOC and ABCC algorithms at Round 1 are shown in Table 4. The minimum, average, and standard deviation of the objective values (Equ. (8)) are also 
Table 4 Objective values of PSOC, GSOC and ABCC algorithms at Round 1

\begin{tabular}{lllll}
\hline No. of nodes & Clustering & \multicolumn{3}{c}{ Objective value } \\
\cline { 3 - 5 } Sink node) & methods & Minimum & Average & $\begin{array}{c}\text { Standard } \\
\text { deviation }\end{array}$ \\
\hline \multirow{3}{*}{$100(0,0)$} & PSOC & 0.000241 & 0.000263 & 0.000012 \\
& GSOC & 0.000241 & 0.000260 & 0.000009 \\
& ABCC & 0.000241 & 0.000250 & 0.000005 \\
\hline \multirow{3}{*}{$100($ Center $)$} & PSOC & 0.000119 & 0.000208 & 0.000016 \\
& GSOC & 0.000119 & 0.000208 & 0.000013 \\
& ABCC & 0.000119 & 0.000208 & 0.000014 \\
\hline \multirow{3}{*}{$200(0,0)$} & PSOC & 0.000516 & 0.000653 & 0.000026 \\
& GSOC & 0.000515 & 0.000604 & 0.000022 \\
& ABCC & 0.000514 & 0.000586 & 0.000024 \\
\hline \multirow{3}{*}{$200($ Center $)$} & PSOC & 0.000264 & 0.000278 & 0.000011 \\
& GSOC & 0.000260 & 0.000276 & 0.000004 \\
& ABCC & 0.000258 & 0.000271 & 0.000004 \\
\hline \multirow{3}{*}{$400(0,0)$} & PSOC & 0.004236 & 0.005747 & 0.000532 \\
& GSOC & 0.003993 & 0.005693 & 0.000410 \\
& ABCC & 0.003872 & 0.005535 & 0.000610 \\
\hline \multirow{2}{*}{$400($ Center $)$} & PSOC & 0.000602 & 0.000681 & 0.000034 \\
& GSOC & 0.000593 & 0.000680 & 0.000022 \\
& ABCC & 0.000593 & 0.000680 & 0.000027 \\
\hline
\end{tabular}

reported in this table. The objective values for the networks with the sink at the center are better than those with the sink at $(0,0)$. The experimental results obtained by the three swarm intelligent algorithms, namely PSOC, GSOC and ABCC, are illustrated in Tables 4 and 5. The performance of GSOC is better than that of PSOC, while the ABCC algorithm achieved the best solutions for all networks. This may be attributable to the cognitively-inspired hierarchical search strategy in $\mathrm{ABCC}$ matching perfectly with the intrinsic dynamics in the cognitive wireless sensor networks.

\subsection{Comparisons to related clustering algorithms}

We use the same performance measures to compare the clustering methods for 100, 200, and 400-node networks as shown in Figures 8, 10 and 11. The overall performance of ABCC is also compared to LEACH, LEACH-C, and HEED in Tables 6, 7, and 8. The proposed ABCC algorithm provides the best/optimal network configuration at each round in terms of both the number and assignment of cluster heads. In contrast, LEACH and HEED only use a non-optimal number of cluster heads, specifying it as a percentage of the total number of nodes in each round. Heinzelman $[10,11]$ validated the performance of their methodology. LEACH-C is better than LEACH for all networks with the exception of the 200 -node network with the sink at $(0,0)$. The performance 


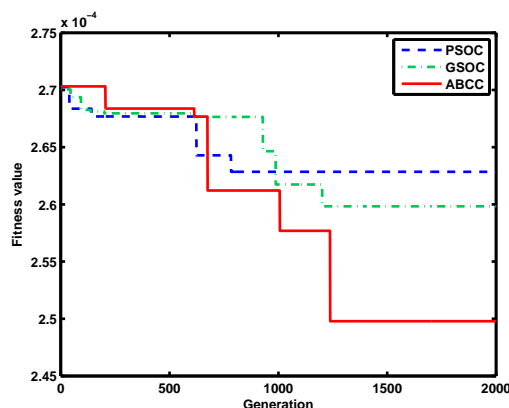

(a) 100 nodes with Sink $(0,0)$

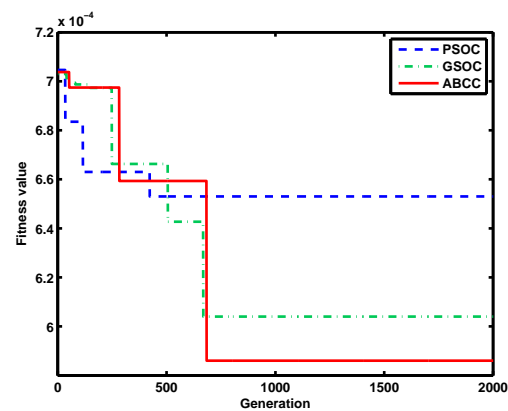

(c) 200 nodes with Sink $(0,0)$

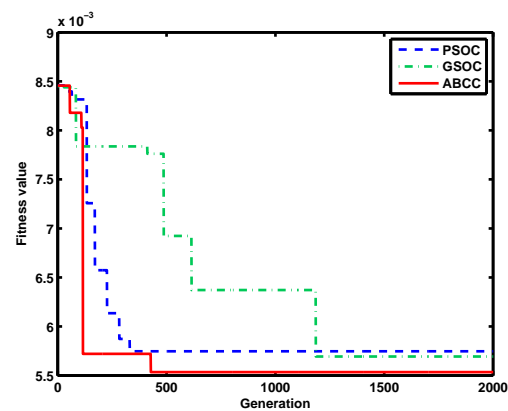

(e) 400 nodes with Sink $(0,0)$

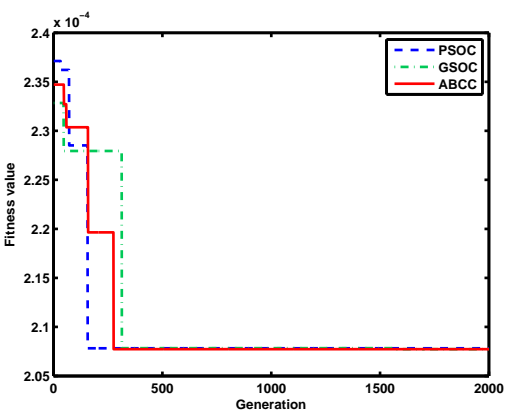

(b) 100 nodes with Sink (Center of nodes)

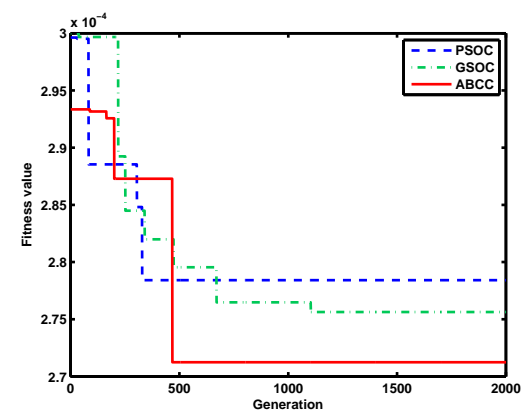

(d) 200 nodes with Sink (Center of nodes)

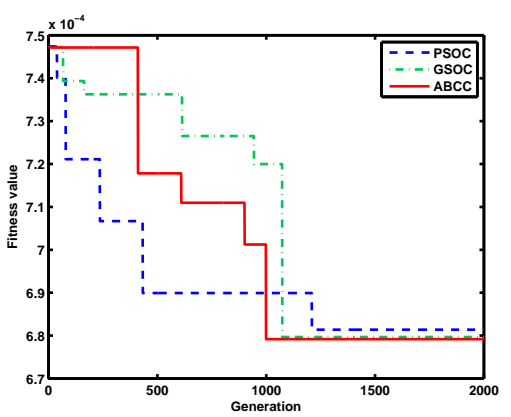

(f) 400 nodes with Sink (Center of nodes)

Fig. 7 Rates of convergence using PSOC, GSOC and ABCC algorithms at Round 1

of the HEED algorithm is variable. HEED has good performance compared to LEACH and LEACH-C for the 100 and 200-node networks with sinks at their center, but achieves the worst results for the 200-node network with a sink at $(0,0)$ and the 400-node network for both sink locations. The performance of $\mathrm{ABCC}$ is consistently better than the other clustering methods.

With reference to Figure 8 and Table 6 it can be seen that the clustering design solutions with center sink using HEED and ABCC are better than the 
Table 5 Comparisons of performance using PSOC, GSOC and ABCC algorithms

\begin{tabular}{|c|c|c|c|c|}
\hline $\begin{array}{l}\text { No. of nodes } \\
\text { (Sink node) }\end{array}$ & $\begin{array}{l}\text { Clustering } \\
\text { methods }\end{array}$ & $\begin{array}{r}\text { Round } \\
\text { first node dies }\end{array}$ & $\begin{array}{r}\text { Round } \\
\text { last node dies }\end{array}$ & $\begin{array}{r}\text { Total data } \\
\text { messages received }\end{array}$ \\
\hline \multirow{3}{*}{$100(0,0)$} & PSOC & 4275 & 4348 & 428890 \\
\hline & GSOC & 4386 & 4431 & 436273 \\
\hline & $\mathrm{ABCC}$ & 4429 & 4443 & 439378 \\
\hline \multirow{3}{*}{100 (Center) } & PSOC & 6607 & 6614 & 654632 \\
\hline & GSOC & 6612 & 6615 & 654860 \\
\hline & $\mathrm{ABCC}$ & 6612 & 6616 & 654979 \\
\hline \multirow{3}{*}{$200(0,0)$} & PSOC & 2188 & 3032 & 583947 \\
\hline & GSOC & 2463 & 3213 & 590219 \\
\hline & $\mathrm{ABCC}$ & 2904 & 3336 & 600930 \\
\hline \multirow{3}{*}{200 (Center) } & PSOC & 4204 & 4285 & 812429 \\
\hline & GSOC & 4216 & 4302 & 843317 \\
\hline & $\mathrm{ABCC}$ & 4302 & 4310 & 857349 \\
\hline \multirow{3}{*}{$400(0,0)$} & PSOC & 406 & 5327 & 555692 \\
\hline & GSOC & 523 & 6029 & 556461 \\
\hline & $\mathrm{ABCC}$ & 560 & 6681 & 560474 \\
\hline \multirow{3}{*}{400 (Center) } & PSOC & 1548 & 3180 & 1041952 \\
\hline & GSOC & 1912 & 3189 & 1089373 \\
\hline & $\mathrm{ABCC}$ & 2212 & 3204 & 1103129 \\
\hline
\end{tabular}

Table 6 Comparisons of performance using clustering methods for 100-node networks

\begin{tabular}{llrrr}
\hline $\begin{array}{l}\text { No. of nodes } \\
\text { (Sink node) }\end{array}$ & $\begin{array}{l}\text { Clustering } \\
\text { methods }\end{array}$ & $\begin{array}{r}\text { Round } \\
\text { first node dies }\end{array}$ & $\begin{array}{r}\text { Round } \\
\text { last node dies }\end{array}$ & $\begin{array}{r}\text { Total data } \\
\text { messages received }\end{array}$ \\
\hline \multirow{4}{*}{$100(0,0)$} & LEACH & 3506 & 3900 & 383430 \\
& LEACH-C & 4170 & 4271 & 414946 \\
& HEED & 3689 & 4430 & 432562 \\
& ABCC & 4429 & 4443 & 439378 \\
\hline \multirow{5}{*}{100 (Center) $)$} & LEACH & 3584 & 4180 & 404222 \\
& LEACH-C & 4307 & 4332 & 428429 \\
& HEED & 6612 & 6797 & 669816 \\
& ABCC & 6612 & 6616 & 654979 \\
\hline
\end{tabular}

other methods for the 100 node network. Figure 9 shows the differences in active (living) nodes (black circles) and dead nodes (white circles) for each clustering method at Rounds 4000 and 6620 for the 100 node center sink network. The first dead node is at Round 3584. 24 nodes have expired by Round 4000, and all nodes have died by Round 4180 using LEACH. The first dead node was at Round 4307, and all nodes died by Round 4332 using LEACH-C. Most of nodes remain active using HEED and ABCC with the first dead nodes only occurring at Round 6612. There is only one dead node using HEED and 3 dead nodes using ABCC, while all nodes are dead by Round 6620 using LEACH and LEACH-C. 


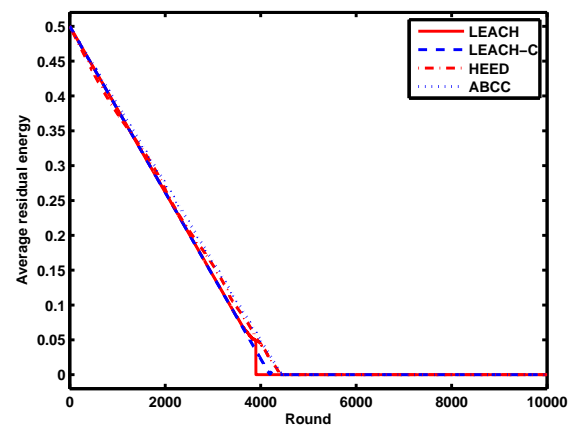

(a) 100 nodes with Sink $(0,0)$

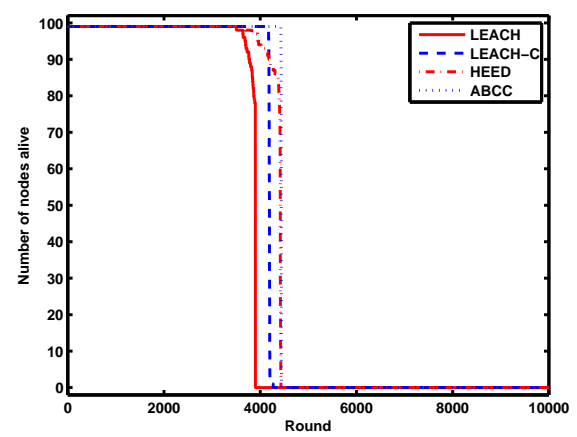

(c) 100 nodes with Sink $(0,0)$

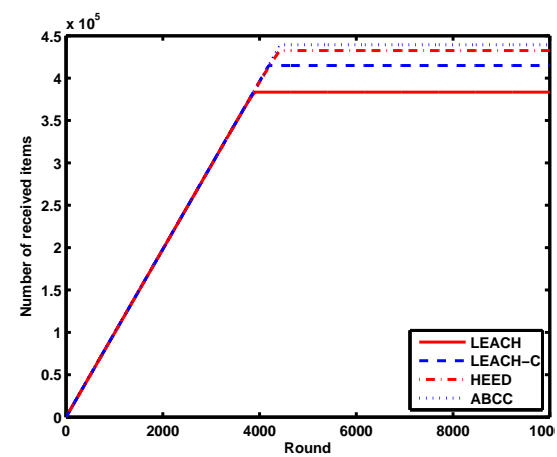

(e) 100 nodes with Sink $(0,0)$

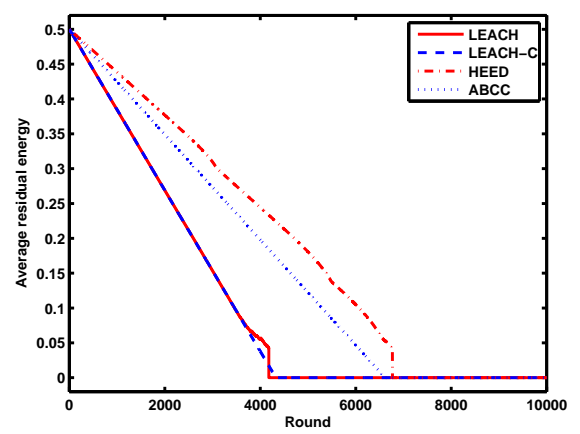

(b) 100 nodes with Sink (Center of nodes)

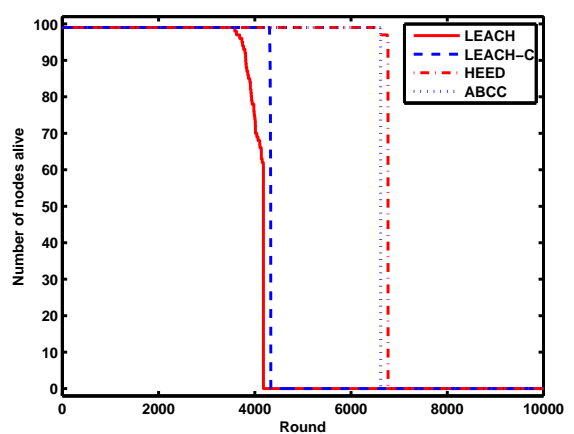

(d) 100 nodes with Sink (Center of nodes)

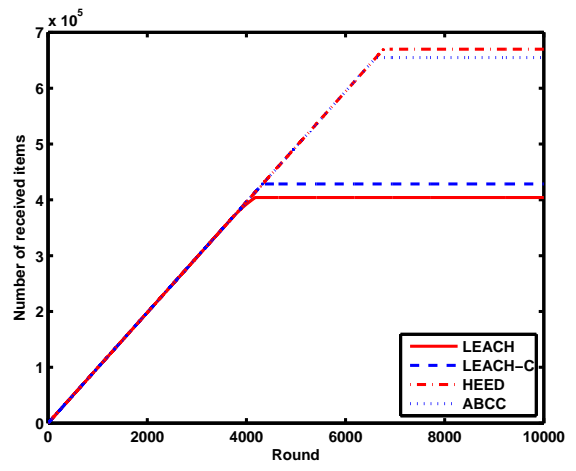

(f) 100 nodes with Sink (Center of nodes)

Fig. 8 Performance comparisons for 100-node networks

A comparison of the performance of the clustering methods for the different sink locations considered is provided in Figure 10 and Table 7 for the 200 node network. The performance of the ABCC algorithm is generally better than that of LEACH, LEACH-C, and HEED. The clustering design solution obtained with ABCC when the sink is centrally located is the best for the 200 


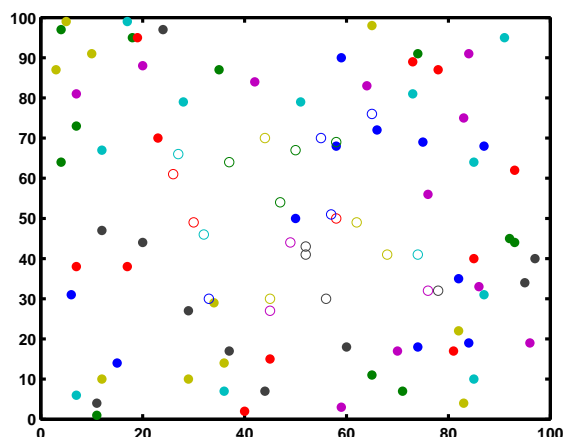

(a) LEACH Round 4000

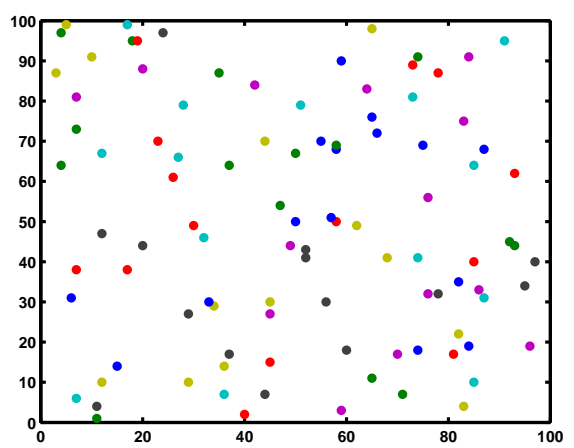

(c) LEACH-C Round 4000

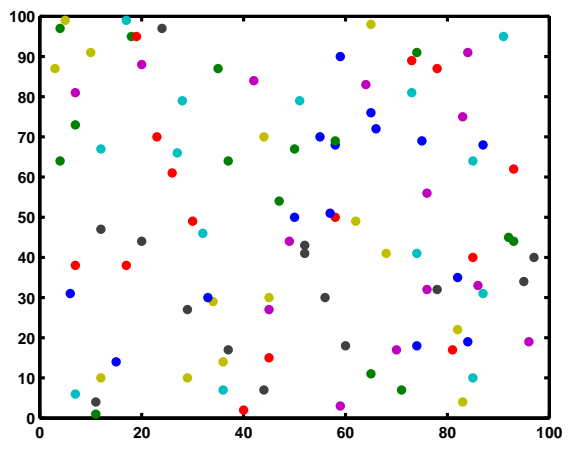

(e) HEED Round 4000

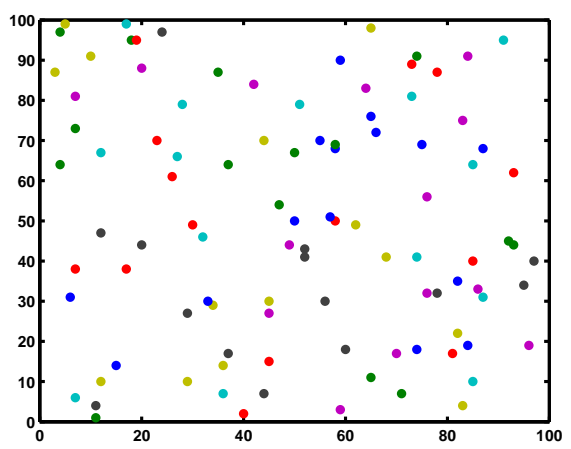

(g) ABCC Round 4000

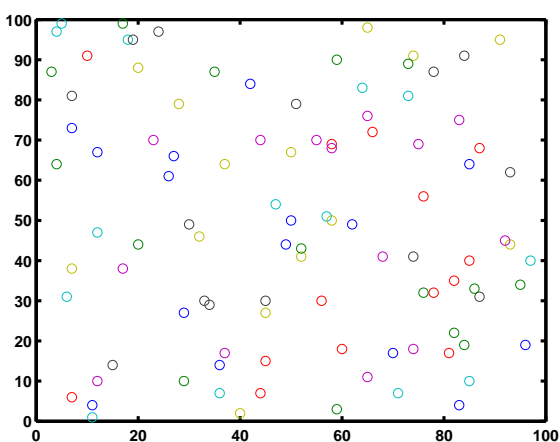

(b) LEACH Round 6620

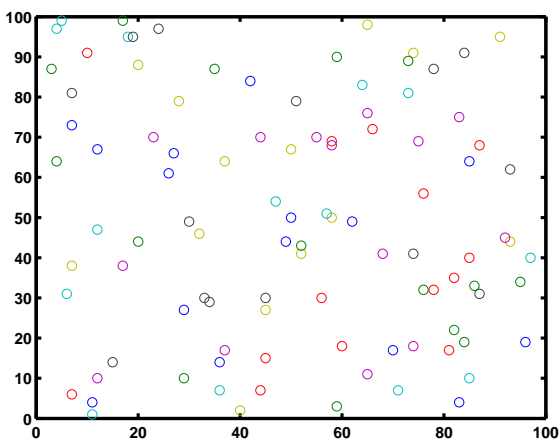

(d) LEACH-C Round 6620

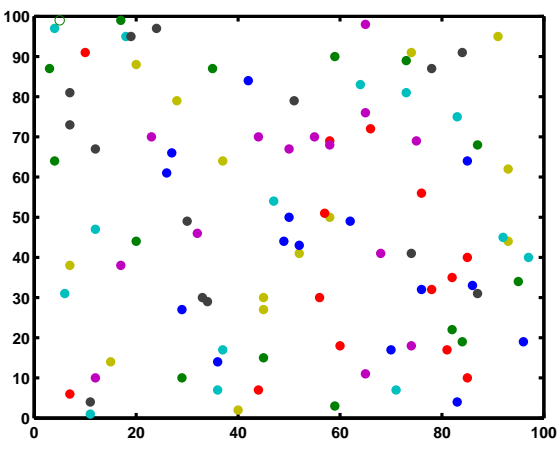

(f) HEED Round 6620

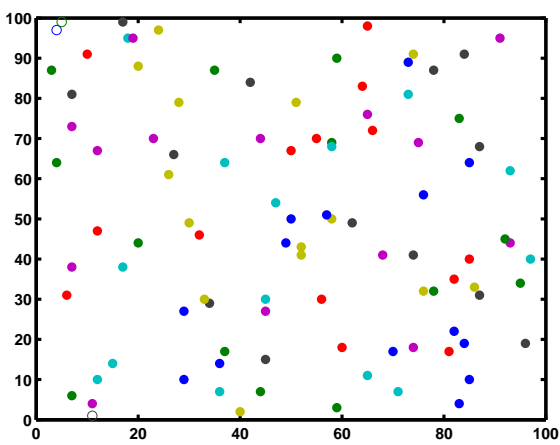

(h) ABCC Round 6620

Fig. 9 Alive node (black circle) and dead node (white circle) for the 100 nodes with Sink (Center of nodes) 


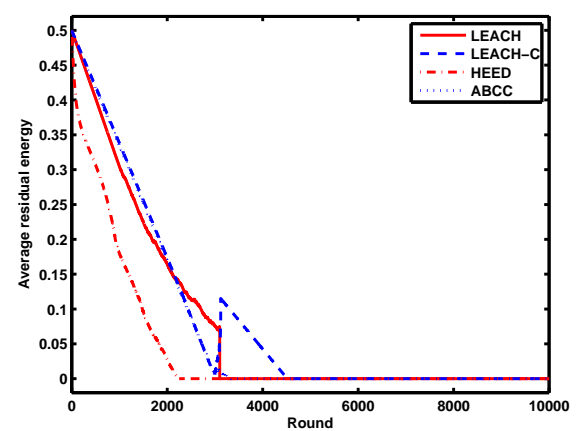

(a) 200 nodes with $\operatorname{Sink}(0,0)$

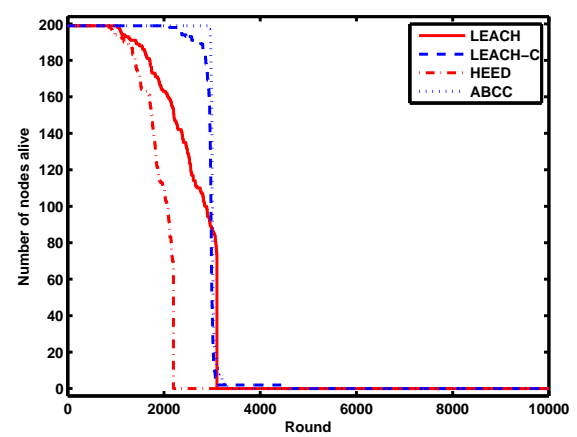

(c) 200 nodes with Sink $(0,0)$

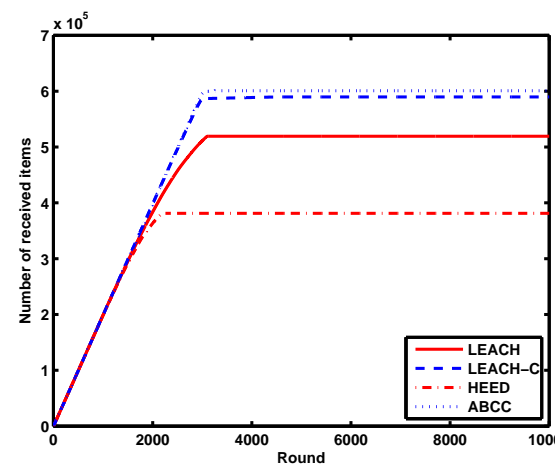

(e) 200 nodes with $\operatorname{Sink}(0,0)$

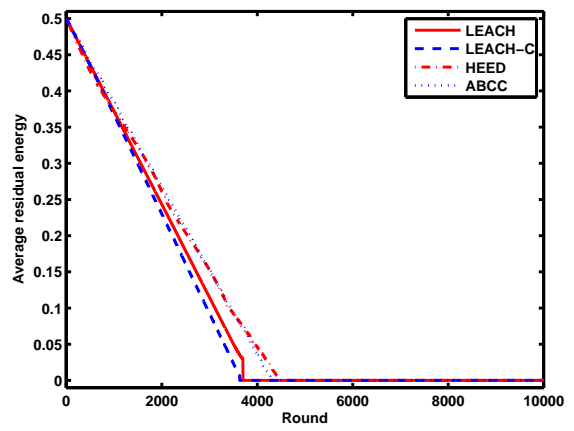

(b) 200 nodes with Sink (Center of nodes)

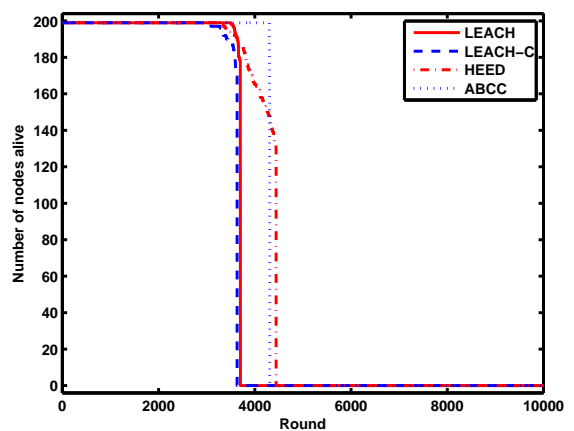

(d) 200 nodes with Sink (Center of nodes)

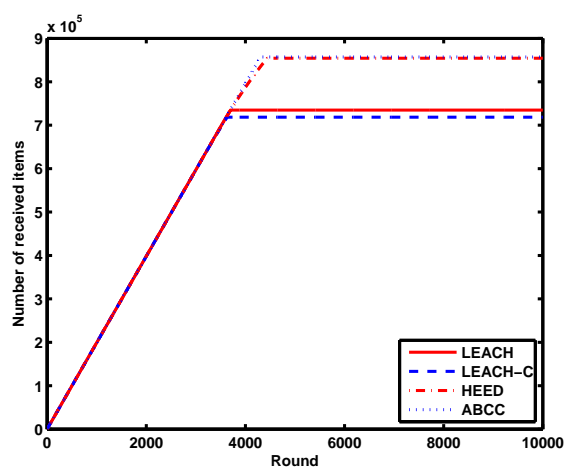

(f) 200 nodes with Sink (Center of nodes)

Fig. 10 Performance comparisons for 200-node networks

node network. The first node dies at Round 4,302, and the last node dies at round 4,310 . The total data messages received is 857,349 . HEED is the worst performing method when the sink is located at $(0,0)$.

A similar pattern is observed for the 400 node networks with different sink locations, as shown in Figure 11 and Table 8, with ABCC substantially better 
Table 7 Comparisons of performance using clustering methods for 200-node networks

\begin{tabular}{llrrr}
\hline $\begin{array}{l}\text { No. of nodes } \\
\text { (Sink node) }\end{array}$ & $\begin{array}{l}\text { Clustering } \\
\text { methods }\end{array}$ & $\begin{array}{r}\text { Round } \\
\text { first node dies }\end{array}$ & $\begin{array}{r}\text { Round } \\
\text { last node dies }\end{array}$ & $\begin{array}{r}\text { Total data } \\
\text { messages received }\end{array}$ \\
\hline \multirow{3}{*}{$200(0,0)$} & LEACH & 1030 & 3100 & 519376 \\
& LEACH-C & 2119 & 4517 & 589751 \\
& HEED & 855 & 2198 & 381256 \\
& ABCC & 2904 & 3336 & 600930 \\
\hline \multirow{5}{*}{$200($ Center $)$} & LEACH & 3508 & 3700 & 734792 \\
& LEACH-C & 3011 & 3632 & 718482 \\
& HEED & 3285 & 4444 & 854271 \\
& ABCC & 4302 & 4310 & 857349 \\
\hline
\end{tabular}

Table 8 Comparisons of performance using clustering methods for 400-node networks

\begin{tabular}{llrrr}
\hline $\begin{array}{l}\text { No. of nodes } \\
\text { (Sink node) }\end{array}$ & $\begin{array}{l}\text { Clustering } \\
\text { methods }\end{array}$ & $\begin{array}{r}\text { Round } \\
\text { first node dies }\end{array}$ & $\begin{array}{r}\text { Round } \\
\text { last node dies }\end{array}$ & $\begin{array}{r}\text { Total data } \\
\text { messages received }\end{array}$ \\
\hline \multirow{4}{*}{$400(0,0)$} & LEACH & 46 & 2640 & 395594 \\
& LEACH-C & 317 & 2858 & 554136 \\
& HEED & 94 & 1407 & 302591 \\
& ABCC & 560 & 6681 & 560474 \\
\hline \multirow{4}{*}{400 (Center) } & LEACH & 888 & 3540 & 1041461 \\
& LEACH-C & 1390 & 2763 & 1040614 \\
& HEED & 843 & 2485 & 799151 \\
& ABCC & 2212 & 3204 & 1103129 \\
\hline
\end{tabular}

than LEACH, LEACH-C, and HEED. Again, the clustering design solution with the Sink located at the center yields the best results.

\section{Conclusions}

The paper presented a novel cognitively-inspired algorithm, namely the artificial bee colony clustering (ABCC) algorithm, for the optimal configuration of cognitive wireless sensor networks. Network configuration is specified via a binary decision variable assigned to each node signifying it as either a cluster head or sensor node. Once cluster heads are specified, sensor nodes are assigned on a nearest cluster head basis.

We use an objective function in the ABCC algorithm to reach the minimization of a weighted combination of the average dissipated energy and the standard deviation of residual energy of the nodes in CWSNs, and seek to prolong network lifetime. Hence, the clustering solutions obtained using ABCC are optimal in the sense of conserving and balancing energy consumption. In contrast, the alternative CWSN clustering methods considered (LEACH, LEACH-C and HEED), are sub-optimal in this regard as they randomly select cluster heads from the available nodes. A further advantage of the ABCC al- 


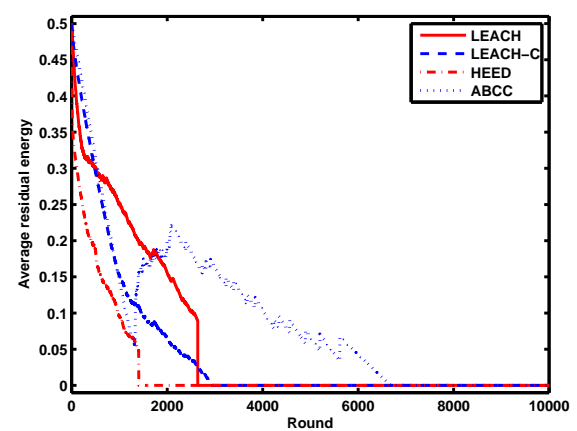

(a) 400 nodes with $\operatorname{Sink}(0,0)$

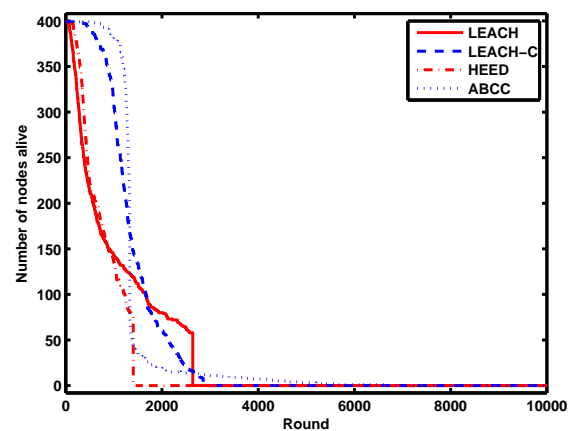

(c) 400 nodes with Sink $(0,0)$

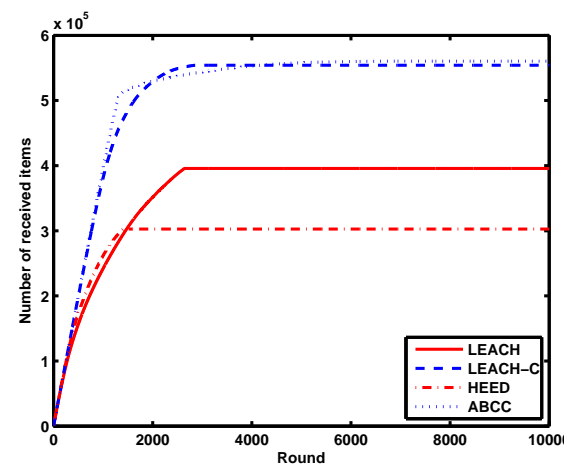

(e) 400 nodes with $\operatorname{Sink}(0,0)$

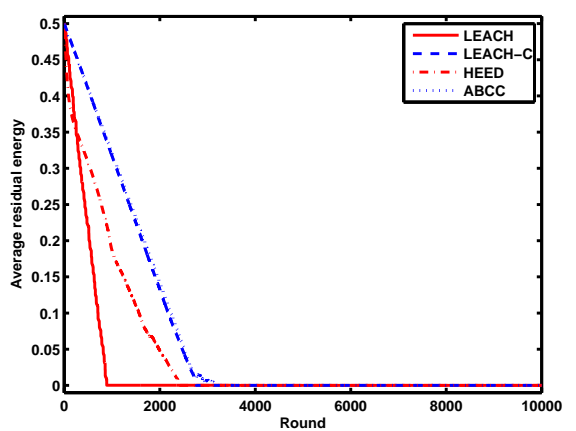

(b) 400 nodes with Sink (Center of nodes)

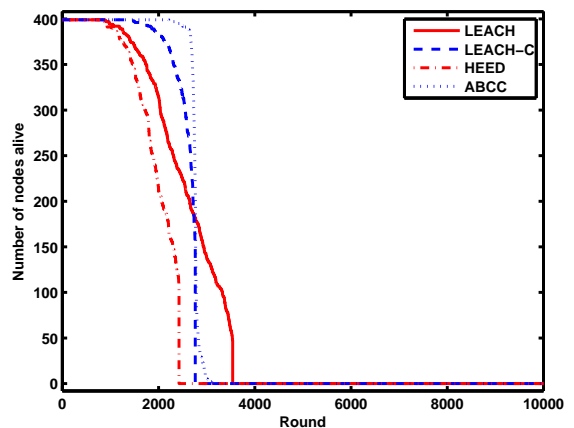

(d) 400 nodes with Sink (Center of nodes)

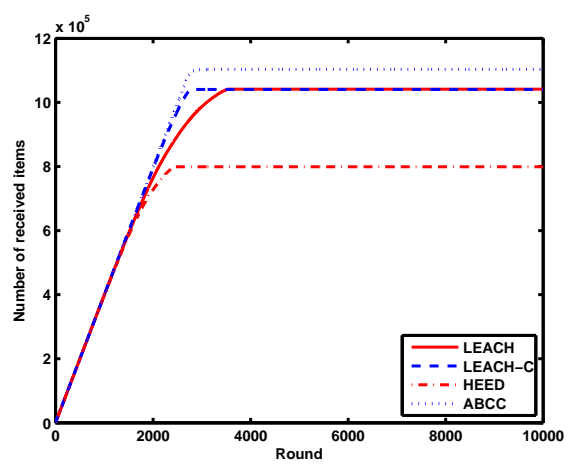

(f) 400 nodes with Sink (Center of nodes)

Fig. 11 Comparisons of performance measures for 400 node network

gorithm is that it has fewer tuning parameters than other heuristic algorithms employed with CWSNs, leading to a more robust optimisation methodology.

The experimental results illustrated that the clustering designs with the sink located at the centre of the network are better than those with the origin as the sink. The proposed ABCC algorithm is generally better than LEACH, LEACH-C, and HEED and is substantially better than these alternatives as 
the number of nodes in the CWSN increases. In effect the ABCC algorithm optimally coincides with the dynamics of the cluster heads and sensor nodes and can adapt to topological changes to any network graph over time.

As demonstrated in this paper, the cognitively-inspired ABCC algorithm is an effective approach to optimally configuring cognitive wireless sensor networks. As future work, we will compare more systematically the ABCC algorithm with closely related existing approaches, such as the stochastic diffusion search algorithm, to further reveal the advantages and disadvantages of the considered algorithms. It is also of interest to explore any subsequent theoretical analysis of these cognitively-inspired algorithms.

Acknowledgements The authors sincerely thank the editors and anonymous reviewers for their helpful suggestions on how to improve the presentation of our paper. This study is supported by 2016 Research Grant from Kangwon National University (Grant No. 520160235) and the Program for New Century Excellent Talents in University (Grant No. NCET-11$0861)$.

\section{Compliance with Ethical Standards}

Conflict of interest Sung-Soo Kim, Ji-Hwan Byeon, Seokcheon Lee, Sean McLoone and Hongbo Liu declare that they have no conflict of interest.

Informed Consent All procedures followed were in accordance with the ethical standards of the responsible committee on human experimentation (institutional and national) and with the Declaration of Helsinki 1975, as revised in 2008 (5). Additional informed consent was obtained from all patients for which identifying information is included in this article.

Human and Animal Rights This article does not contain any studies with human participants or animals performed by any of the authors.

\section{References}

1. Abbasi, A., Younis, M.: A survey on clustering algorithms for wireless sensor networks. Computer Communications 30(14), 2826-2841 (2007)

2. Abdullah, A., Hussain, A., Khan, I.H.: Introduction: Dealing with big data-lessons from cognitive computing. Cognitive Computation 7(6), 635-636 (2015)

3. Aslam, M., Javaid, N., Rahim, A., Nazir, U., Bibi, A., Khan, Z.: Survey of extended leach-based clustering routing protocols for wireless sensor networks. In: Proceedings of IEEE 14th International Conference on High Performance Computing and Communication \& IEEE 9th International Conference on Embedded Software and Systems, pp. 1232-1238. IEEE (2012)

4. Bishop, J.: Stochastic searching networks. In: Proceedings of the 1st IEEE Conference on Artificial Neural Networks, pp. 329-331. IEEE, London (1989)

5. Dechene, D., El Jardali, A., Luccini, M., Sauer, A.: A survey of clustering algorithms for wireless sensor networks. Tech. rep., Department of Electrical and Computer Engineering, The University Of Western Ontario (2006)

6. Ding, S., Zhang, J., Jia, H., Qian, J.: An adaptive density data stream clustering algorithm. Cognitive Computation 8(1), 30-38 (2016)

7. Dubey, H.M., Pandit, M., Panigrahi, B.: A biologically inspired modified flower pollination algorithm for solving economic dispatch problems in modern power systems. Cognitive Computation 7(5), 594-608 (2015)

8. Fernández-Caballero, A., González, P., Navarro, E.: Cognitively-inspired computing for gerontechnology. Cognitive Computation 8(2), 297-298 (2016) 
9. He, S., Wu, Q., Saunders, J.: Group search optimizer: An optimization algorithm inspired by animal searching behavior. IEEE Transactions on Evolutionary Computation 13(5), 973-990 (2009)

10. Heinzelman, W.B., Chandrakasan, A.P., Balakrishnan, H.: An application-specific protocol architecture for wireless microsensor networks. IEEE Transactions on Wireless Communications 1(4), 660-670 (2002)

11. Heinzelman, W.R., Chandrakasan, A., Balakrishnan, H.: Energy-efficient communication protocol for wireless microsensor networks. In: Proceedings of the 33rd Annual Hawaii International Conference on System Sciences, pp. 1-10. IEEE (2000)

12. Hunt, S., Meng, Q., Hinde, C., Huang, T.: A consensus-based grouping algorithm for multi-agent cooperative task allocation with complex requirements. Cognitive Computation 6(3), 338-350 (2014)

13. Ibriq, J., Mahgoub, I.: Cluster-based routing in wireless sensor networks: issues and challenges. In: Proceedings of 2004 Symposium on Performance Evaluation of Computer Telecommunication Systems, pp. 759-766 (2004)

14. Karaboga, D., Basturk, B.: On the performance of artificial bee colony (ABC) algorithm. Applied Soft Computing 8(1), 687-697 (2008)

15. Karaboga, D., Okdem, S., Ozturk, C.: Cluster based wireless sensor network routings using artificial bee colony algorithm. In: Proceedings of 2010 International Conference on Autonomous and Intelligent Systems, pp. 1-5. IEEE (2010)

16. Karaboga, D., Okdem, S., Ozturk, C.: Cluster based wireless sensor network routing using artificial bee colony algorithm. Wireless Networks 18(7), 847-860 (2012)

17. Kennedy, J., Eberhart, R.: Particle swarm optimization. In: Proceedings of 1995 IEEE International Conference on Neural Networks, vol. 4, pp. 1942-1948. IEEE (1995)

18. Kim, S.S., Byeon, J.H., Liu, H., Abraham, A., McLoone, S.: Optimal job scheduling in grid computing using efficient binary artificial bee colony optimization. Soft Computing $\mathbf{1 7}(5), 867-882$ (2013)

19. Kulkarni, R., Forster, A., Venayagamoorthy, G.: Computational intelligence in wireless sensor networks: A survey. Communications Surveys \& Tutorials, IEEE 13(1), 68-96 (2011)

20. Li, G., Niu, P., Xiao, X.: Development and investigation of efficient artificial bee colony algorithm for numerical function optimization. Applied Soft Computing 12(1), 320-332 (2012)

21. Li, J., Pan, Q.: Solving the large-scale hybrid flow shop scheduling problem with limited buffers by a hybrid artificial bee colony algorithm. Information Sciences 316, 487-502 (2015)

22. Liu, H., Abraham, A., Clerc, M.: Chaotic dynamic characteristics in swarm intelligence. Applied Soft Computing 7(3), 1019-1026 (2007)

23. Liu, X.: A survey on clustering routing protocols in wireless sensor networks. Sensors $\mathbf{1 2}(8), 11,113-11,153(2012)$

24. Loubière, P., Jourdan, A., Siarry, P., Chelouah, R.: A sensitivity analysis method for driving the artificial bee colony algorithm's search process. Applied Soft Computing (2016)

25. Muth, F., Papaj, D.R., Leonard, A.S.: Bees remember flowers for more than one reason: pollen mediates associative learning. Animal Behaviour 111, 93-100 (2016)

26. Okdem, S., Karaboga, D., Ozturk, C.: An application of wireless sensor network routing based on artificial bee colony algorithm. In: Evolutionary Computation (CEC), 2011 IEEE Congress on, pp. 326-330. IEEE (2011)

27. Ozturk, C., Hancer, E., Karaboga, D.: Dynamic clustering with improved binary artificial bee colony algorithm. Applied Soft Computing 28, 69-80 (2015)

28. al Rifaie, M., Bishop, J.: Stochastic diffusion search review. Journal of Behavioural Robotics 4(3), 155-173 (2013)

29. al Rifaie, M.M., Bishop, J.M., Caines, S.: Creativity and autonomy in swarm intelligence systems. Cognitive Computation 4(3), 320-331 (2012)

30. Salim, A., Osamy, W., Khedr, A.M.: IBLEACH: Intra-Balanced LEACH protocol for wireless sensor networks. Wireless Networks 20(6), 1515-1525 (2014)

31. Siddique, N., Adeli, H.: Nature inspired computing: An overview and some future directions. Cognitive Computation 7(6), 706-714 (2015) 
32. Song, L., Hatzinakos, D.: Cognitive networking of large scale wireless systems. International Journal of Communication Networks and Distributed Systems 2(4), 452-475 (2009)

33. Ullah, A., Li, J., Hussain, A., Yang, E.: Towards a biologically inspired soft switching approach for cloud resource provisioning. Cognitive Computation 8(5), 992-1005 (2016)

34. Yang, X.S., Cui, Z., Xiao, R., Gandomi, A.H., Karamanoglu, M.: Swarm Intelligence and Bio-inspired Computation: Theory and Applications. Elsevier (2013)

35. Ye, D., Chen, Z.: A new approach to minimum attribute reduction based on discrete artificial bee colony. Soft Computing 19(7), 1893-1903 (2015)

36. Younis, O., Fahmy, S.: Heed: a hybrid, energy-efficient, distributed clustering approach for ad hoc sensor networks. IEEE Transactions on Mobile Computing 3(4), 366-379 (2004)

37. Younis, O., Krunz, M., Ramasubramanian, S.: Node clustering in wireless sensor networks: Recent developments and deployment challenges. Network, IEEE 20(3), 20-25 (2006)

38. Yurtkuran, A., Emel, E.: An adaptive artificial bee colony algorithm for global optimization. Applied Mathematics and Computation 217, 1004-1023 (2015) 